\title{
Deficiências e incapacidades na hanseníase: do diagnóstico à alta por cura
}

\author{
Emanuelle Malzac Freire de Santana ${ }^{1}$, \\ Karen Krystine Gonçalves de Brito², \\ Jordana de Almeida Nogueira ${ }^{3}$, \\ Oriana Deyze Correia Paiva Leadebal ${ }^{4}$, \\ Marta Miriam Lopes Costa ${ }^{5}$, \\ Mirian Alves da Silva 6 , \\ Maria Júlia Guimarães Oliveira Soares ${ }^{7}$
}

\section{RESUMO}

Objetivou-se comparar o grau de incapacidade física, os sítios corporais afetados, as deficiências e incapacidades presentes e os nervos acometidos no diagnóstico e na alta em pacientes com hanseníase. Trata-se de um estudo desenvolvido entre 2009 e 2014 em centro de referência para hanseníase na Paraíba. Envolveu 414 prontuários, utilizando formulário estruturado. Os dados foram analisados através de técnicas de estatística descritiva (frequência absoluta e percentagem) e inferencial (Teste de Wilcoxon e Mcnemar). Nota-se decréscimo do acometimento dos sítios corporais (nariz $p=0,000$ ), das deficiências (ressecamento $p=0,002$ e ferida $p=0,000$ no nariz e úlcera $p=0,004$ nos pés) e da quantidade de nervos afetados $(p=0,000)$ entre o diagnóstico e a alta por cura. $\mathrm{Na}$ análise dos anos 2009-2014 observa-se redução da quantidade de pacientes apresentando grau de incapacidade física 2. Conclui-se, portanto, que mesmo após a alta os pacientes estão propícios a desenvolver ou agravar incapacidades físicas, necessitando de acompanhamento periódico.

Descritores: Hanseníase; Pessoas com Deficiência; /prevenção \& controle; Atenção Secundária à Saúde.

\footnotetext{
${ }^{1}$ Fisioterapeuta, Mestre em Enfermagem. Discente do Programa de Pós-Graduação em Enfermagem, nível Doutorado, da Universidade Federal da Paraíba. Docente Supervisora da Faculdade Maurício de Nassau - João Pessoa. João Pessoa, PB, Brasil. E-mail: manumalzac@gmail.com.

2 Enfermeira, Mestre em Enfermagem. Discente do Programa de Pós-Graduação em Enfermagem, nível Doutorado, da Universidade Federal da Paraíba. João Pessoa, PB, Brasil. E-mail: karen enf@yahoo.com.br.

${ }^{3}$ Enfermeira, Doutora em Enfermagem. Professora Associada da Universidade Federal da Paraíba. João Pessoa, PB, Brasil. E-mail: jalnogueira31@gmail.com.

${ }^{4}$ Enfermeira, Doutora em Enfermagem. Professora Adjunta da Universidade Federal da Paraíba. João Pessoa, PB, Brasil. E-mail: orianadcpl@gmail.com.

${ }^{5}$ Enfermeira, Doutora em Sociologia. Professora Associada da Universidade Federal da Paraíba. João Pessoa, PB, Brasil. E-mail: marthamiryam@ @ hotmail.com.

${ }^{6}$ Enfermeira, Doutora em Enfermagem. Professora Adjunta da Universidade Federal da Paraíba. João Pessoa, PB, Brasil. E-mail: miads.enf@gmail.com.

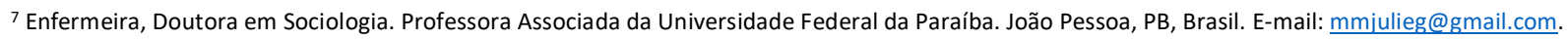

\section{Como citar esse artigo:}

Santana EMF, Brito KKG, Nogueira JA, Leabedal ODCP, Costa MML, Silva MA, et al. Deficiências e incapacidades na hanseníase: do diagnóstico à alta por cura. Rev. Eletr. Enf. [Internet]. 2018 [acesso em:

];20:v20a15. Disponível em: https://doi.org/10.5216/ree.v20.50436. 


\section{INTRODUÇÃO}

A hanseníase é uma doença infecciosa de alto poder incapacitante que dispõe de diferentes formas de apresentação clínica. A restrita relação entre o Mycobacterium Leprae e a resposta imune celular do hospedeiro são determinantes na gravidade da doença, embora sua predileção pelas células da pele e nervos periféricos seja cientificamente comprovada ${ }^{(1)}$.

Basicamente, os mecanismos causadores das deficiências e incapacidades na hanseníase podem ocorrer através das vias neurogênica e inflamatória. Déficits sensitivos, motores e autonômicos são classificados como causas primárias, enquanto lesões traumáticas, retrações e infecções pós-traumáticas são consideradas secundárias, visto que ocorrem em decorrência da ausência de cuidados preventivos após o processo primário(2).

Nos primeiros estágios, ocorre acometimento da sensibilidade térmica, seguida de redução até perda da sensibilidade dolorosa e tátil. $\mathrm{O}$ dano neural está presente nas fases mais avançadas podendo ocasionar parestesias e plegias musculares ${ }^{(1)}$. Esses comprometimentos podem tornar os indivíduos propensos a acidentes, queimaduras, feridas e amputações, sendo responsáveis pelo surgimento de sequelas permanentes ${ }^{(3)}$.

Geralmente, o início ocorre de forma insidiosa e sem sintomatologia e, em muitos casos, pode explicar o atraso no seu diagnóstico ${ }^{(1)}$, considerado fator de risco para o desenvolvimento das incapacidades. O diagnóstico tardio e a falta de tratamento adequado da hanseníase podem resultar em diminuição da capacidade laborativa, restrição à participação social e danos psicológicos ${ }^{(4)}$.

As deficiências são descritas como perda ou anormalidade de uma estrutura ou função psicológica, fisiológica ou anatômica, enquanto as incapacidades são consideradas consequências das deficiências no que se refere ao rendimento funcional para desempenhar uma atividade, sendo determinadas de acordo com o contexto ambiental e social no qual o indivíduo está inserido, posto as diferentes percepções culturais, atitudes, disponibilidade de serviços e legislação(5).

Dados da Organização Mundial de Saúde evidenciam que aproximadamente 14.000 novos casos da doença foram diagnosticados com incapacidade de grau 2 (IG2) no cenário mundial em 2015. Destes, 1.752 foram registrados apenas no Brasil, responsável por aproximadamente $89 \%$ de todos os casos com IG2 nas Américas ${ }^{(6)}$. No âmbito estadual, estudo sobre a análise epidemiológica da hanseníase na Paraíba verificou que no momento do diagnóstico 3,92\% dos casos já apresentavam IG2(7).

Frente aos aspectos fisiopatológicos da doença e em consonância com a epidemiologia supracitada, é indispensável avaliar a integridade da função neural e classificar o grau de incapacidade física (GIF) entre os indivíduos com hanseníase tanto no diagnóstico quanto na alta por cura. Esse monitoramento deve ser realizado para evitar ou minimizar a progressão do dano neural, além de prevenir possíveis sequelas.

No Brasil, estudos que envolvem as incapacidades físicas dos pacientes com hanseníase são, em sua maioria, descritivos ou pontuais, não se detendo a análises temporais, evolutivas ou comparativas ${ }^{(8-9)}$. Diante deste déficit de dados, estudos sobre prevalência, tipologia e tendenciosidade das deficiências e incapacidades para monitoramento da magnitude da doença e fomento de estratégias de prevenção aos agravos são pertinentes.

Partindo do pressuposto que aproximadamente $20 \%$ de todos os novos casos de pacientes com hanseníase apresentam algum grau de incapacidade física no momento do diagnóstico e que em torno de $23 \%$ vão 
desenvolver incapacidades mesmo após a alta ${ }^{(3,10)}$, sentiu-se a necessidade de comparar a situação clínica dos pacientes no que concerne às incapacidades físicas no momento do diagnóstico e da alta por cura.

Nessa perspectiva, o objetivo deste estudo foi comparar o grau de incapacidade física, os principais sítios corporais afetados, as deficiências e incapacidades presentes e os nervos acometidos nos momentos do diagnóstico e da alta por cura.

\section{MÉTODO}

Trata-se de um estudo de caráter descritivo, retrospectivo, base populacional e fonte documental com delineamento quantitativo realizado entre 2009 a 2014 com prontuários de pacientes com diagnóstico de hanseníase atendidos em um centro de referência especializado para o tratamento da doença no Estado da Paraíba/PB.

A população do estudo foi composta por 485 prontuários de pacientes da região metropolitana de João Pessoa. Como critérios de inclusão foram instituídos: prontuários de pacientes que iniciaram e concluíram o tratamento para hanseníase no centro de referência e que possuíam preenchida a ficha de avaliação simplificada das funções neurais e complicações no diagnóstico e na alta por cura. Estabeleceu-se como critérios de exclusão: prontuários de pacientes com comprometimento cognitivo $(n=4)$; prontuários com informações incompletas relacionadas a avaliação simplificada das funções neurais e complicações e determinação do GIF ( $n=61$ ); e prontuários de pacientes que não realizaram o tratamento no tempo preconizado pelo Ministério da Saúde (MS) $(n=6)$. Desta forma, a amostra do estudo foi composta por 414 prontuários.

Os dados foram coletados por meio de instrumento estruturado a partir dos prontuários dos pacientes, onde foram extraídas variáveis sociodemográficas (sexo, idade e grau de escolaridade) e clínico epidemiológicas (classificação operacional - paucibacilar ou multibacilar, formas clínicas - indeterminada, tuberculóide, neural pura, dimorfa e virchowiana).

O instrumento também foi composto por informações referentes ao momento do diagnóstico e da alta por cura provenientes da ficha de avaliação simplificada das funções neurais e complicações. A utilização desta ficha é preconizada pelo MS e baseia-se em uma avaliação neurológica simplificada para identificar a gravidade das lesões presentes nos olhos, nariz, mãos e pés ${ }^{(11)}$, sendo parte integrante da avaliação realizada no serviço.

Em cada ficha, foi considerado o grau máximo de incapacidades físicas variando de zero (0) até dois (2). A graduação zero é utilizada quando não há nenhum dano presente nos olhos, mãos e pés, o grau um (1) corresponde a diminuição ou perda da sensibilidade e o grau dois (2) quando há presença de deformidades visíveis devido a hanseníase ${ }^{(12)}$. É preconizado pelo MS que o GIF seja avaliado no momento do diagnóstico, na ocorrência de estados reacionais e na alta por cura(11).

Salienta-se que em 2016 foi lançado um manual técnico-operacional intitulado "Diretrizes para vigilância, atenção e eliminação da hanseníase como problema de saúde pública" em que os critérios para avaliação do GIF foram modificados passando a incluir a força muscular como critério para determinação deste ${ }^{(11)}$. Todavia, como o estudo refere-se aos anos de 2009 a 2014 essa alteração não foi considerada.

Os dados obtidos foram codificados para tabulação no aplicativo Microsoft Excel e em seguida exportados e analisados pelo software Statistical Package for the Social Science (SPSS) versão 20. Foram realizadas técnicas 
de estatística descritiva para obtenção da frequência absoluta e percentagem e inferencial através dos testes de Wilcoxon e McNemar, utilizados para comparação das diferenças entre duas amostras relacionadas, sendo o primeiro utilizado para variáveis ordinais e o segundo em variáveis nominais. Adotou-se nível de significância de $5 \%(p<0,05)$.

No processo de investigação foram adotadas as observâncias éticas contempladas nas diretrizes e normas regulamentadoras para pesquisa envolvendo seres humanos - Resolução 466/12 do Conselho Nacional de Saúde, principalmente no que diz respeito ao sigilo e à confidencialidade dos dados. O projeto foi aprovado pelo Comitê de Ética em Pesquisa do Centro de Ciências da Saúde da Universidade Federal da Paraíba sob protocolo 443/14, CAAE 34284414.3.0000.5188.

\section{RESULTADOS}

Na caracterização sociodemográfica e clínica da população, 243 (59\%) eram homens, 115 (27,8\%) estavam na faixa etária de 31 a 45 anos, 222 (53,6\%) possuíam ensino fundamental como grau de escolaridade (53,6\%) e 229 (55,3\%) eram procedentes do município de João Pessoa. Com relação as características clínicas, 251 (61\%) foram classificados como multibacilares e 145 (35\%) possuíam forma clínica dimorfa.

Ao comparar o GIF no momento do diagnóstico e na alta por cura não foi encontrada diferença estatisticamente significativa $(p=0,148)$, todavia percebe-se melhora deste indicador, tendo em vista o aumento na quantidade de pacientes classificados como grau $0(+3,2 \%)$ e redução de indivíduos no grau $1(-2,9 \%)$ (Tabela 1).

Tabela 1: Comparação do GIF no diagnóstico e na alta por cura entre pacientes com hanseníase residentes na região metropolitana de João Pessoa entre 2009 a 2014 (n=414). João Pessoa, PB, Brasil, 2016.

\begin{tabular}{cccc}
\hline GIF & Diagnóstico $\mathbf{n}(\%)$ & Alta $\mathbf{n}(\%)$ & p-valor $^{(\mathbf{a})}$ \\
\hline 0 & $246(59,4)$ & $259(62,6)$ & \\
1 & $122(29,5)$ & $110(26,6)$ & $\mathrm{p}=0,148$ \\
2 & $46(11,1)$ & $45(10,9)$ & \\
\hline
\end{tabular}

Legenda: ${ }^{(a)}$ Teste de Wilcoxon

Na Figura 1, é possível observar a evolução temporal do GIF entre 2009 a 2014. Tanto no diagnóstico como na alta, ocorreu expressiva mudança no GIF entre os anos de 2009 e 2010 com considerável aumento do GIF 0 e declínio dos GIFs 1 e 2. Nos anos subsequentes, ocorreram oscilações e manutenção dos graus com ênfase para redução de IG2 no ano de 2014. Observa-se também que entre 2011 e 2013 ocorreu aumento do GIF 1.

No que tange aos sítios corporais, nota-se decréscimo de seu comprometimento ao comparar o diagnóstico e a alta por cura com diferença estatisticamente significativa apenas para o nariz $(p=0,000)$ (Tabela 2).

A Tabela 3 lista as principais deficiências encontradas, evidenciando redução na maioria destas na comparação do diagnóstico e da alta por cura com diferença estatisticamente significativa para ressecamento $(p=0,002)$ e ferida $(p=0,000)$ no nariz e úlcera $(p=0,004)$ nos pés. Verificou-se ainda um aumento das deficiências consideradas mais graves nas mãos (garra rígida e reabsorção) e nos pés (garra rígida, reabsorção e pé caído), embora sem relevância estatística. 
Figura 1: Evolução temporal do GIF no diagnóstico e na alta por cura de pacientes com hanseníase residentes na região metropolitana de João Pessoa entre 2009 a 2014.

\section{Diagnóstico x Alta}

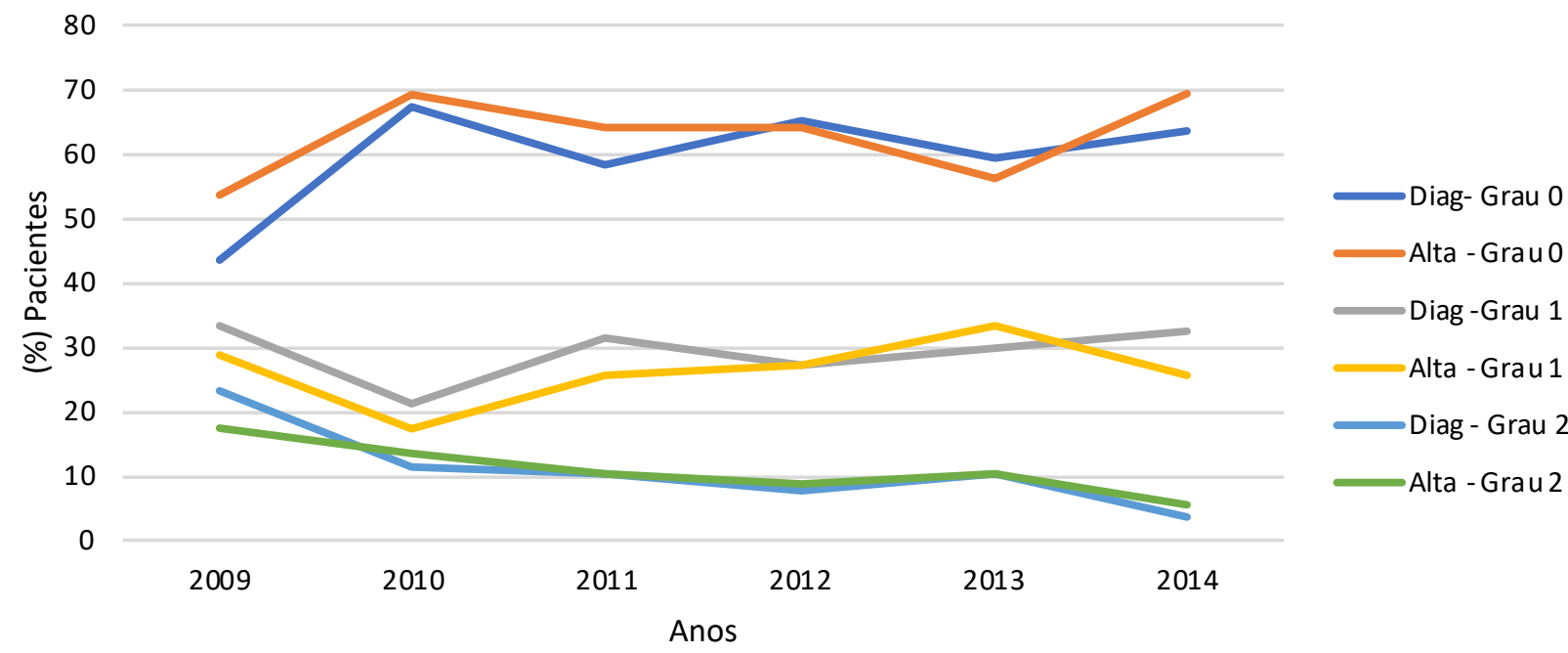

Tabela 2: Comparação dos sítios corporais afetados no diagnóstico e na alta por cura entre pacientes com hanseníase residentes na região metropolitana de João Pessoa entre 2009 a 2014 ( $n=414)$. João Pessoa, PB, Brasil, 2016.

\begin{tabular}{cccc}
\hline Sítio corporal afetado & Diagnóstico & Alta & p-valor $^{(\mathbf{b})}$ \\
\hline Olhos & 28 & 19 & $\mathrm{p}=0,137$ \\
Nariz & 47 & 18 & $\mathrm{p}=0,000^{*}$ \\
Mãos & 58 & 52 & $\mathrm{p}=0,429$ \\
Pés & 154 & 143 & $\mathrm{p}=0,178$ \\
\hline
\end{tabular}

Legenda: ${ }^{(b)}$ Teste de McNemar / * p-valor $<0,05$ (resultado significativo)

Tabela 3: Comparação das principais deficiências presentes no diagnóstico e na alta por cura entre pacientes com hanseníase residentes na região metropolitana de João Pessoa entre 2009 a 2014 ( $n=414$ ). João Pessoa, PB, Brasil, 2016.

\begin{tabular}{|c|c|c|c|}
\hline Tipos de deficiências & Diagnóstico & Alta & p-valor ${ }^{(b)}$ \\
\hline \multicolumn{4}{|l|}{ Olhos } \\
\hline Ectrópio & 2 & 1 & $p=1,000$ \\
\hline Diminuiç̧ão da sensibilidade da córnea & 16 & 8 & $p=0,096$ \\
\hline Opacidade da córnea & 7 & 5 & $p=1,000$ \\
\hline Acuidade visual & 7 & 8 & $P=1,000$ \\
\hline \multicolumn{4}{|l|}{ Nariz } \\
\hline Ressecamento & 37 & 17 & $p=0,002^{*}$ \\
\hline Ferida & 21 & 2 & $p=0,000^{*}$ \\
\hline \multicolumn{4}{|l|}{ Mãos } \\
\hline Hipoestesia & 53 & 48 & $p=0,522$ \\
\hline Úlcera & 17 & 9 & $p=0,115$ \\
\hline Garra Móvel & 13 & 12 & $p=1,000$ \\
\hline Garra Rígida & 2 & 4 & $p=0,500$ \\
\hline Reabsorção & 3 & 6 & $p=0,250$ \\
\hline \multicolumn{4}{|l|}{ Pés } \\
\hline Hipoestesia & 152 & 138 & $p=0,088$ \\
\hline Úlcera & 21 & 9 & $p=0,004^{*}$ \\
\hline Garra Móvel & 2 & 2 & $p=1,000$ \\
\hline Garra Rígida & 0 & 1 & $p=1,000$ \\
\hline Reabsorção & 1 & 2 & $p=1,000$ \\
\hline Pé caído & 9 & 10 & $p=1,000$ \\
\hline
\end{tabular}

Legenda: ${ }^{(b)}$ Teste de McNemar / * $p$-valor $<0,05$ (resultado significativo) 
No que diz respeito ao número de nervos afetados (Tabela 4), foi encontrada média de 1,10 $( \pm 1,45)$ nervos afetados no momento do diagnóstico e 0,80 $( \pm 1,30)$ na alta, constatando-se diferença estatisticamente significativa $(p=0,000)$. É possível observar diminuição do comprometimento de todos os nervos do diagnóstico para alta apresentando diferença estatisticamente significativa, exceto para o nervo mediano $(p=0,056)$.

Tabela 4: Comparação dos nervos afetados no diagnóstico e na alta por cura entre pacientes com hanseníase residentes na região metropolitana de João Pessoa entre 2009 a 2014 (n=414). João Pessoa, PB, Brasil, 2016.

\begin{tabular}{cccc}
\hline Nervos Afetados & Diagnóstico & Alta & $\mathbf{p}_{\text {-valor }}$ (b) $^{(2)}$ \\
\hline Ulnar & 118 & 84 & $\mathrm{p}=0,001^{*}$ \\
Radial & 60 & 34 & $\mathrm{p}=0,002^{*}$ \\
Mediano & 95 & 76 & $\mathrm{p}=0,056$ \\
Fibular & 83 & 56 & $\mathrm{p}=0,005^{*}$ \\
Tibial Posterior & 102 & 80 & $\mathrm{p}=0,034^{*}$ \\
\hline
\end{tabular}

Legenda: ${ }^{(b)}$ Teste de McNemar / * $p$-valor $<0,05$ (resultado significativo)

\section{DISCUSSÃO}

Verificou-se neste estudo, elevada porcentagem de indivíduos apresentando IG1 e IG2 tanto no diagnóstico $(29,5 \%$ e $11,1 \%)$ quanto alta por cura $(26,6 \%$ e $10,9 \%)$. Outras pesquisas também apontam esse panorama endêmico para a região Nordeste ${ }^{(10,13)}$, classificada como a terceira região brasileira com maior coeficiente geral de deteç̧ão de casos (23,8/100.000 habitantes), configurando-se área de alta endemicidade e importante na manutenção da transmissão da doença(14).

Apesar destes índices e das diferenças regionais relacionadas à carga da hanseníase, de maneira geral, o Brasil vem apresentando tendência de queda do IG2 ao longo dos anos, observando-se declínio de $29 \%$ nos últimos 10 anos (2005-2015) ${ }^{(6)}$. Esta situação também no presente estudo, através da linha decrescente na análise da evolução temporal dos casos com IG2 principalmente entre os anos de 2013 e 2014.

Em comparação aos países asiáticos, o Brasil possui menores proporções de IG2 ${ }^{(6)}$, o que pode ser reflexo da ampliação da assistência na atenção básica em saúde proporcionando melhorias dos serviços referentes ao controle da doença ${ }^{(15)}$.

Ressalta-se que as IG2 são utilizadas para compor indicadores epidemiológicos de avaliação do Programa Nacional de Controle da Hanseníase, sendo utilizadas na mensuração da força de morbidade, magnitude e perfil epidemiológico da doença, além de avaliar a qualidade e a efetividade dos serviços de saúde prestados ${ }^{(16)}$.

A diminuição da ocorrência de casos novos com IG2 no momento do diagnóstico sugere menor diagnóstico tardio, que quando acompanhada do declínio da taxa de deteç̧ão caracteriza diminuição da magnitude da endemia ao longo dos anos ${ }^{(11)}$.

Esses achados corroboram com as atividades propostas pelo "Plano integrado de ações estratégicas de eliminação da hanseníase, filariose, esquistossomose e oncocercose como problema de saúde pública, tracoma como causa de cegueira e controle das geohelmintíases: plano de ação 2011-2015", lançado pelo MS com o propósito de erradicar ou reduzir de forma drástica a carga desses agravos e, se tratando da hanseníase, enfatiza o aumento da deteç̧ão precoce e da cura dos casos diagnosticados ${ }^{(17)}$.

Tendo em vista a carga da hanseníase no Brasil e no mundo e visando reduzí-la ainda mais, a atual estratégia de combate à doença proposta pela Organização Mundial de Saúde tem como uma de suas metas para o ano de 
2020 a redução da taxa de indivíduos com IG2 para <1 caso por milhão de habitantes. Dentre os pilares recomendados pela estratégia, está o combate a hanseníase e suas complicações, visando a detecção precoce mediante a busca ativa de casos, o início imediato/adesão ao tratamento e a melhora da prevenção e do manejo das incapacidades ${ }^{(18)}$.

Nesta perspectiva, salienta-se a importância da atuação de uma equipe multiprofissional para prevenir o surgimento/agravamento das incapacidades físicas, destacando-se a atuação do enfermeiro e do fisioterapeuta. O enfermeiro, através da consulta de enfermagem, é responsável pelo cuidado mensal do paciente com hanseníase, devendo supervisionar a administração da medicação, identificar sinais e sintomas da doença, orientar sobre a realização do autocuidado e encaminhar o paciente para outros profissionais quando necessário(19). O fisioterapeuta, além de atuar na fase de prevenção das incapacidades, em que são fornecidas orientações sobre a doença, suas complicações e o autocuidado, realiza a reabilitação das sequelas ocasionadas pela doença ${ }^{(20)}$.

Na análise dos sítios corporais, o decréscimo do seu comprometimento, evidenciado neste estudo, também foi verificado por outros autores ${ }^{(21-22)}$. Todavia, constatou-se que apenas o nariz apresentou relevância estatística, o que corrobora com a diminuição do ressecamento e da presença de feridas encontradas neste sítio.

Apesar de não serem quantificadas para determinação do GIF, as lesões no nariz apresentam importância tanto no aspecto físico devido as deformidades, quanto no psicológico pelo estigma associado à doença, posto que este é considerado determinante nas relações interpessoais.

Além de comprometer o indivíduo em sua imagem corporal, o comprometimento desta região também é capaz de alterar de forma parcial ou total a função respiratória interferindo na realização de atividades laborais e sociais $^{(22)}$. Com base nessas premissas, bem como, na facilidade de observação, intui-se que houve maior atenção com essa área.

Considerando o cenário das deficiências, merece destaque a diminuição da presença de úlceras cutâneas na região plantar. Complicação frequente na hanseníase, o mal perfurante plantar é decorrente de alterações na sensibilidade e motricidade do pé resultando em perdas sensitivas e fraqueza/paralisia da musculatura, respectivamente. Em sua maioria, estas lesões estão localizadas nas áreas responsáveis pelo suporte de peso interferindo na funcionalidade e na qualidade de vida dos indivíduos ${ }^{(23)}$.

Esse achado evidencia a importância da atuação da fisioterapia no acompanhamento de pacientes com hanseníase, e em se tratando da terapêutica das úlceras cutâneas, tem como finalidade a estimulação do processo de cicatrização da lesão, reduzindo seu tempo de exposição e proporcionando ao indivíduo retorno mais breve as suas atividades diárias ${ }^{(24)}$. Dentre as condutas executadas no serviço, é possível citar: utilização do laser de baixa intensidade, realização de exercícios terapêuticos, reeducação da marcha e prescrição de próteses e órteses, configurando a fisioterapia como uma importante ferramenta para o tratamento das consequências da hanseníase.

Embora tenha sido observada redução da maioria das deficiências, é importante frisar que no momento da alta ocorreu aumento da quantidade de indivíduos apresentando complicações mais severas nas mãos (garra rígida e reabsorção) e nos pés (garra rígida, reabsorção e pé caído), regiões mais comprometidas. Essas manifestações são secundárias ao comprometimento dos nervos periféricos e poderiam ter sido evitadas se o 
diagnóstico e o tratamento fossem realizados de maneira precoce, enquanto o bacilo estivesse limitado às terminações nervosas superficiais.

Ainda no que diz respeito às deficiências, é possível observar a identificação de menores índices de comprometimento a nível ocular quando comparado as demais regiões. Este fato pode ser explicado pela informação "NR" (não realizado) registrada em algumas fichas, principalmente nos itens acuidade visual e sensibilidade corneana. Os recursos materiais limitados são fatores que configuram impedimento para a prevenção de incapacidades ${ }^{(25)}$, e podem ter influenciado na realização da avaliação desta região.

Analisando os troncos nervosos, com exceção do nervo mediano, ocorreu redução do comprometimento neural. Estudo realizado em Centro Nacional de Referência para o tratamento da hanseníase na cidade de Uberlândia/MG também identificou redução do acometimento nervoso na comparação do diagnóstico versus alta, com média de 2,5 nervos afetados por paciente no diagnóstico e 2,2 na alta ${ }^{(22)}$. Todavia, esses resultados foram superiores aos encontrados neste estudo.

Ressalta-se que como consequência da lesão neural podem surgir queimaduras, ferimentos, úlceras, fissuras, atrofias, articulações rígidas e desequilíbrio muscular ${ }^{(2)}$, tornando-se imprescindível que os pacientes sejam orientados a autoavaliar diariamente seus sítios corporais e realizar atividades de autocuidado visando prevenir o surgimento das incapacidades ou evitar seu agravamento. Neste ínterim, é possível citar como atividades: hidratar e lubrificar a pele, cuidar das unhas e das calosidades, inspecionar calçados antes de usá-los, usar palmilhas especiais e calçados adaptados, evitar transitar em ambientes escuros e com desníveis de pisos, dentre outras ${ }^{(23)}$.

Tendo em vista as implicações do acometimento dos troncos nervosos, consubstancia-se a importância dos achados clínicos deste estudo, posto que sua redução evita o desencadeamento de sequelas físicas. Contudo, embora tenha ocorrido significativa diminuição do comprometimento nervoso, chama atenção a quantidade de pacientes que continuaram com nervos afetados após a alta.

No período pós-alta, a prevenção e o manejo de incapacidades residuais ainda constituem um desafio, haja vista que após os pacientes concluírem o tratamento estes são excluídos do registro ativo e orientados pelos profissionais de saúde a retornarem ao serviço de acordo com as suas necessidades, não recebendo, portanto, acompanhamento de forma sistemática. Neste sentido, a integração dos serviços da rede de atenção à saúde é de fundamental importância para monitorar esses pacientes após a alta por cura, evitando a instalação de sequelas permanentes.

Torna-se relevante discutir ainda sobre as lacunas encontradas nos prontuários no que se refere a avaliação do GIF, o que evidencia uma fragilidade do serviço, tendo em vista que os dados da avaliação do GIF são utilizados para compor indicadores operacionais do MS que monitoram o progresso da eliminação da hanseníase enquanto problema de saúde pública, além de mensurarem a qualidade do atendimento nos serviços de saúde ${ }^{(11)}$.

A situação se agrava pelo fato do cenário da pesquisa ser um centro de referência para o tratamento da hanseníase no Estado da Paraíba que pressupõe equipe qualificada, estruturada e equipamento ideais para a implementação de políticas públicas de saúde.

Outro aspecto dos prontuários que merece destaque foi o registro da presença de atrofia do primeiro espaço interósseo/musculatura interóssea da mão, sinal característico de lesão do nervo ulnar. 
No período deste estudo as atrofias musculares ainda não eram consideradas para classificação do GIF, passando a ser incorporadas apenas nas diretrizes para vigilância, atenção e eliminação da hanseníase como problema de saúde pública publicadas pelo MS no ano de 2016. Na atual classificação, pacientes com atrofias musculares são considerados com IG2(11).

Cabe ainda destacar que nas novas diretrizes a força muscular também foi inserida na determinação do GIF, de modo que pacientes com força muscular reduzida que antes não eram classificados em nenhum grau passaram a ser grau um (1). Essa mudança permite monitoramento mais abrangente e consequente maior controle dos pacientes, visto que o dano neural também ocasiona prejuízos na função motora.

Com a incorporação dessas modificações relacionadas ao GIF, muito provavelmente, o quadro epidemiológico exposto sofreria uma mudança em seu contexto, visto o aumento da quantidade de indivíduos que passariam a apresentar os graus um (1) e dois (2) de incapacidade física. O panorama atual é formulado com base na subnotificação destes graus, visto que alguns prontuários já discriminavam a presença de incapacidades, antes não consideradas na avaliação preconizada pelo MS.

Esse dado corrobora a importância das mudanças propostas, bem como, para o fomento de novas pesquisas, a partir desse momento, que terão maior força e embasamento para demonstrar a real situação no que condiz a efetividade e a qualidade dos serviços de atendimento ao indivíduo acometido pela hanseníase.

\section{CONCLUSÃO}

De maneira geral constatou-se melhora do GIF, redução do comprometimento de todos os sítios corporais bem como da maioria das deficiências e diminuição do acometimento e da quantidade de nervos afetados ao comparar o momento do diagnóstico à alta por cura. Esses achados revelam que a assistência realizada no centro de atenção secundária à saúde no que concerne à prevenção de incapacidades e a reabilitação dos indivíduos se mostrou satisfatória, apesar de lacunas em atividades previstas pelas políticas direcionadas aos indivíduos com hanseníase terem sido identificadas.

A análise da evolução temporal do GIF no recorte de 2009-2014 também demonstra progresso, posto que ao longo dos anos ocorreu redução da quantidade de pacientes apresentando IG2 tanto no diagnóstico quanto na alta por cura, o que pode estar relacionado à redução do diagnóstico tardio e rapidez no serviço de referência, apesar do cenário da pesquisa se tratar de um centro de atenção secundária à saúde e, portanto, não realizar a busca ativa dos doentes, embora exista uma rede de atenção à saúde.

Apesar dos aspectos positivos mencionados, é importante enfatizar que uma expressiva parcela de pacientes recebeu alta do tratamento medicamentoso apresentando nervos afetados e/ou incapacidades de grau um (1) ou dois (2), necessitando, desta forma, de um acompanhamento periódico para prevenir o surgimento/agravamento de deformidades, o que nos remete a refletir sobre a necessidade de mudanças na política vigente destinada a essa população, como a criação de um protocolo de seguimento dos indivíduos após a alta da poliquimioterapia.

O estudo possui limitações devido a fragilidade encontrada nos prontuários no que diz respeito as informações incompletas referentes a avaliação simplificada das funções neurais e complicações e determinação do GIF, que acarretaram prejuízos no delineamento da real situação da hanseníase na região estudada. 
Levando em consideração as mudanças propostas pelo MS a partir do ano de 2016 e a escassez de estudos que abordem a temática das incapacidades, sugere-se a realização de novas pesquisas, seja em centros de referências ou em âmbito municipal, como forma de monitorar os progressos alcançados na busca pela eliminação da hanseníase e orientar ações relacionadas a prevenção, diagnóstico, tratamento e reabilitação das sequelas físicas ocasionadas pela doença.

\section{REFERÊNCIAS}

1. Araújo AERA, Aquino DMC, Goulart IMB, Pereira SRF, Figueiredo IA, Serra HO, et al. Neural complications and physical disabilities in leprosy in a capital of northeastern Brazil with high endemicity. Rev Bras Epidemiol [Internet]. 2014 [acesso em: 17 ago. 2018];17(4):899-910. Disponível em: http://doi.org/10.1590/1809-4503201400040009.

2. Rosa GR, Lima MM, Brito WI, Moreira AM. Análise da completude do grau de incapacidade em hanseníase da Regional de Saúde de Rondonópolis/MT. Revista Gestão \& Saúde [Internet]. 2016 [acesso em: 17 ago. 2018];7(1):82-95. Disponível em: http://dx.doi.org/10.18673/gs.v7i1.22068.

3. Ribeiro GC, Lana FCF. Incapacidades físicas em hanseníase: caracterização, fatores relacionados e evolução. Cogitare Enferm. [Internet]. 2015 [acesso em: 17 ago. 2018];20(3):496-503. Disponível em: http://dx.doi.org/10.5380/ce.v20i3.41246. 4. Buna ATM, Rocha FCG, Alves EM, Granja FBC, Sousa DJ, Silva MGP. Incapacidades físicas nos pacientes com hanseníase cadastrados em uma unidade de saúde de São Luís - MA. Revista Interdisciplinar [Internet] 2015 [acesso em: 17 ago. 2018];8(1):115-22. Disponível em: https://revistainterdisciplinar.uninovafapi.edu.br/index.php/revinter/article/view/579. 5. Malta DC, Stopa SR, Canuto R, Gomes NL, Mendes VLF, Goulart BN, et al. Self-reported prevalence of disability in Brazil, according to the National Health Survey, 2013. Cien Saude Colet [Internet] 2016 [acesso em: 17 ago. 2018];21(10):3253-64. Disponível em: https://doi.org/10.1590/1413-812320152110.17512016.

6. World Health Organization. Global leprosy update, 2015: time for action, accountability and inclusion. Wkly Epidemiol Rec [Internet]. 2015 [acesso em: 17 ago. 2018];91(35): 405-20. Disponível em: http://www.who.int/lep/resources/who wer9135/en/. 7. Brito KKG, Andrade SSC, Santana EMF, Matos SDO, Diniz IV, Aguiar ESS, et al. Epidemiological and temporal analysis of leprosy in a Brazilian endemic state. International Archives of Medicine [Internet]. 2016 [acesso em: 17 ago. 2018];9(106):1-8. Disponível em: https://doi.org/10.3823/1977.

8. Oliveira LB, Alves ES, Araújo TME, Melo IV, Araújo RPS, Marques LMF. Epidemiological profile of leprosy in a municipality in the Brazilian Northeast: a retrospective analysis. Revista de Pesquisa: Cuidado é Fundamental Online [Internet]. 2017 [acesso em: 17 ago. 2018];9(3):648-52. Disponível em: https://doi.org/10.9789/2175-5361.2017.v9i3.648-652.

9. Sarmento APA, Pereirao AM, Ribeiro F, Castro JL, Almeida MB, Ramos NM. Perfil epidemiológico da hanseníase no período de 2009 a 2013 no município de Montes Claros (MG). Rev Soc Bras Clin Med [Internet]. 2015 [acesso em: 17 ago. 2018];13(3):180-4. Disponível em: http://files.bvs.br/upload/S/1679-1010/2015/v13n3/a5389.pdf.

10. Pacheco MAB, Aires MLL, Seixas ES. Prevalência e controle de hanseníase: pesquisa em uma ocupação urbana de São Luís, Maranhão, Brasil. Rev Bras Med Fam Comunidade [Internet] 2014 [acesso em: 17 ago. 2018];9(30):23-30. Disponível em: https://doi.org/10.5712/rbmfc9(30)690.

11. Ministério da Saúde, Secretaria de Vigilância em Saúde, Departamento de Doenças Transmissíveis. Diretrizes para vigilância, atenção e eliminação da Hanseníase como problema de saúde publica: manual técnico-operacional [Internet]. Brasília: Ministério da Saúde; 2016 [acesso em: 17 ago. 2018]. Disponível em:

http://portalarquivos2.saude.gov.br/images/pdf/2016/fevereiro/04/diretrizes-eliminacao-hanseniase-4fev16-web.pdf.

12. Neves TV, Souza EB, Valentim IM, Reis IB, Diniz APM, Rocha ESD, et al. Grau de incapacidade física e escore olhos-mãos-e-pés em pacientes hansênicos pós-alta. Revista de APS [Internet]. 2015 [acesso em: 17 ago. 2018];18(3):335-40. Disponível em: https://aps.ufjf.emnuvens.com.br/aps/article/view/2452.

13. Barbosa JC, Ramos Junior AN, Alencar OM, Pinto MSP, Castro CGJ. Atenção pós-alta em hanseníase no Sistema Único de Saúde: aspectos relativos ao acesso na região Nordeste. Cad Saude Colet [Internet]. 2014 [acesso em: 17 ago. 2018];22(4):351-8. Disponível em: https://dx.doi.org/10.1590/1414-462X201400040008.

14. Brito AL, Monteiro LD, Ramos Junior AN, Heukelbach J, Alencar CH. Tendência temporal da hanseníase em uma capital do Nordeste do Brasil: epidemiologia e análise por pontos de inflexão, 2001 a 2012. Rev Bras Epidemiol [Internet]. 2016 [acesso em: 17 ago. 2018];19(1):194-204. Disponível em: https://doi.org/10.1590/1980-5497201600010017.

15. Monteiro LD, Alencar CHM, Barbosa JC, Braga KP, Castro MD, Heukelbach J. Incapacidades físicas em pessoas acometidas pela hanseníase no período pós-alta da poliquimioterapia em um município no Norte do Brasil. Cad Saude Publica [Internet]. 2013 [acesso em: 17 ago. 2018];29(5):909-20. Disponível em: https://doi.org/10.1590/S0102-311X2013000500009.

16. Oliveira KS, Souza J, Zilly A, Silva-Sobrinho RA. Avaliação dos indicadores epidemiológicos e operacionais para a hanseníase em municípios prioritários no estado do Paraná, 2001 a 2010. Epidemiol Serv Saude [Internet]. 2015 [acesso em: 17 ago. 
2018];24(3):507-16. Disponível em: http://www.scielo.br/scielo.php?script=sci arttext\&pid=S2237-

96222015000300507\&lng=en\&nrm=iso\&tlng=pt.

17. Moura LMA, Pereira MA, Veloso LC. Estratégias Utilizadas pelos Serviços de Saúde na Detecção Precoce da Hanseníase: uma Revisão Integrativa. Revista Saúde em Foco [Internet]. 2015 [acesso em: 17 ago. 2018];2(1):130-50. Disponível em:

http://www4.fsanet.com.br/revista/index.php/saudeemfoco/article/view/524.

18. Fine PEM. An important perspective on the recent history of leprosy - and its implications for the current Global Strategy. Lepr Rev [Internet]. 2016 [acesso em: 17 ago. 2018];87(2):146-50. Disponível em:

https://www.lepra.org.uk/Platforms/Lepra/Files/Ir/June16/Editorial_87_2.pdf.

19. Silva LS, Silva TM, Rocha JT, Andrade WG, Lessa EC, Correia NS. A assistência de enfermagem aos portadores de hanseníase assistidos pelo programa de saúde da família. Rev enferm UFPE on line [Internet]. 2016 [acesso em: 17 ago. 2018];10(11):4111-7. Disponível em: https://periodicos.ufpe.br/revistas/revistaenfermagem/article/view/11498/13365.

20. Ferreira JLPM, Cerdeira DQ, Nunes TTV, Guimarães DF, Liberato FRC. Atuação da fisioterapia no acompanhamento de pacientes com hanseníase. Fisioterapia Brasil [Internet]. 2016 [acesso em: 17 ago. 2018];17(5):472-9. Disponível em:

http://portalatlanticaeditora.com.br/index.php/fisioterapiabrasil/article/view/683.

21. Faria CRS, Fregonesi CEPT, Coraza DAG, Andrade DM, Mantovani NADT, Silva JR, et al. Grau de incapacidade física de portadores de hanseníase: estudo de coorte retrospectivo. Arquivos de Ciências da Saúde [Internet]. 2015 [acesso em: 17 ago. 2018];22(4): 5862. Disponível em: https://doi.org/10.17696/2318-3691.22.4.2015.122.

22. Kil AKA, Silvestre CM, Kaminice LM, Quintino LB, Lima LB, Paranhos MB, et al. Deficiências e incapacidades por Hanseníase: Avaliação clínica e epidemiológica dos pacientes atendidos em um Centro de Referência Nacional do Brasil. Hansen Int [Internet]. 2012 [acesso em: 17 ago. 2018];37(1):25-33. Disponível em: http://www.ilsl.br/revista/detalhe artigo.php?id=11777. 23. Pinheiro MGC, Silva SYB, Silva FS, Ataide CAV, Lima IB, Simpson CA. 10.5935/1415-2762.20140066. REME - Rev Min Enferm [Internet]. 2014 [acesso em: 17 ago. 2018];18(4):895-900. Disponível em: https://doi.org/10.5935/1415-2762.20140066. 24. Rosa JM, Torquato SG, Mello D, Dantaas EHM. Relato de experiência sobre o uso do laser de baixa potência no tratamento de úlceras neurotróficas. Rev enferm UFPE on line [Internet]. 2014 [acesso em: 17 ago. 2018];8(5):1330-6. Disponível em: https://periodicos.ufpe.br/revistas/revistaenfermagem/article/view/9817/9997.

25. Gonçalves A. Realities of leprosy control: updating scenarios. Rev Bras Epidemiol [Internet]. 2013 [acesso em: 17 ago. 2018];16(3):611-21. Disponível em: https://doi.org/10.1590/S1415-790X2013000300006. 